\title{
Dynamic morphology and cytoskeletal protein changes during spontaneous inside-out vesiculation of red blood cell membranes
}

\author{
Teresa Tiffert • Virgilio L. Lew
}

Received: 19 October 2013 /Revised: 6 February 2014 / Accepted: 13 February 2014 / Published online: 12 March 2014

(C) The Author(s) 2014. This article is published with open access at Springerlink.com

\begin{abstract}
Vesicle preparations from cell plasma membranes, red blood cells in particular, are extensively used in transport and enzymic studies and in the fields of drug delivery and drug-transport interactions. Here we investigated the role of spectrin-actin, the main components of the red cell cortical cytoskeleton, in a particular mechanism of vesicle generation found to be relevant to the egress process of Plasmodium falciparum merozoites from infected red blood cells. Plasma membranes from red blood cells lysed in ice-cold media of low ionic strength and free of divalent cations spontaneously and rapidly vesiculate upon incubation at $37{ }^{\circ} \mathrm{C}$ rendering high yields of inside-out vesicles. We tested the working hypothesis that the dynamic shape transformations resulted from changes in spectrin-actin configuration within a disintegrating cytoskeletal mesh. We showed that cytoskeletal-free membranes behave like a two-dimensional fluid lacking shape control, that spectrin-actin remain attached to vesiculating membranes for as long as spontaneous movement persists, that most of the spectrin-actin detachment occurs terminally at the time of vesicle sealing and that naked membrane patches increasingly appear during vesiculation. These results support the proposed role of spectrin-actin in spontaneous vesiculation. The implications of these results to membrane dynamics and to the mechanism of merozoite egress are discussed.
\end{abstract}

Keywords Erythrocyte $\cdot$ Erythrocyte membrane . Erythrocyte vesicles $\cdot$ Erythrocyte cytoskeleton $\cdot$ Inside-out vesicles $\cdot$ Merozoite egress

\section{T. Tiffert $(\bowtie) \cdot$ V. L. Lew}

Physiological Laboratory, Department of Physiology, Development and Neuroscience, University of Cambridge, Downing Street, Cambridge CB2 3EG, UK

e-mail: jtt1000@cam.ac.uk

\section{Introduction}

Plasma membrane vesicle preparations of right-side-out (ROVs) and inside-out (IOVs) orientation are a valuable and widely used tool that has contributed a wealth of information on the identity, kinetics and vectorial properties of plasma membrane transporters and enzymes [12, 21, 26, 31, 32, 46]. As with many such tools in biological research, the experimental protocols yielding vesicular preparations were the result of inspired trial and error experimentation, ideas on the mechanism by which vesicles were formed taking shape subsequently. One of the most widely used IOV preparations was that originally developed by Steck and collaborators for red blood cell (RBC) membranes [4, 5, 7, 38-40, 43, 44]. The essential procedural steps were as follows: RBCs were first lysed in many volumes of an ice-cold, hypo-osmotic, lowionic strength medium, free of divalent cations and lightly buffered to $\mathrm{pH}$ 7.5-7.8; the pelleted membranes, usually referred to as "ghosts" at this stage, were further washed and incubated at low temperatures in such media. Final shearing of such treated membranes through thin needles rendered the desired vesicles. One idea about the mechanism of formation of IOVs and ROVs by this method was based on the observation that the conditions caused progressive loss of spectrin and actin, leaving a naked membrane devoid of cytoskeletal support, essentially a giant liposome [38, 40, 44]. Subsequent shearing through thin needles fragmented the membranes into sealed vesicles through fleeting transitional open states of random inside-out or right-side-out topology.

An important property of the vesicles generated by the classic Steck-Kant procedure [43] was the functional preservation of nearly all native enzymes and transporters of the $\mathrm{RBC}$ membrane. The notable exception was the $\mathrm{Ca}^{2+}$-activated $\mathrm{K}^{+}$channel of the RBC membrane (IK1, Kcnn4 [3, 19, 45], also known as the Gardos channel $[13,24,36])$. Searching for the stage in the vesicle-preparation protocol at which channel 
activity was lost, it was discovered that a vesicular preparation with a high yield of IOVs could be generated within 5-10 min of lysis if the membranes were immediately incubated at $37^{\circ} \mathrm{C}$ in the lysis medium [26]. The vesicles generated in this way retained Gardos channel activity $[2,12,26]$.

The rapid generation of such "one-step" vesicles from lysed RBCs enabled the vesiculation process to be followed and recorded under the microscope on a temperaturecontrolled stage [25]. The most surprising finding was that one-step vesicle formation was an entirely spontaneous process; each ghost was seen to transform spontaneously into a bunch of linked vesicles within about $5-8$ min at $37{ }^{\circ} \mathrm{C}$, a process at variance with the giant liposome transitional stage model. A detailed study of this process by a variety of techniques showed that spontaneously generated IOVs were formed following a specific sequence of highly dynamic membrane shape changes. A first critical finding was that the unique lysis conditions leading to vesiculation prevented the sealing of the lytic hole $[25,27,28]$. At $37^{\circ} \mathrm{C}$, the membrane around the lytic hole rapidly curled outwards forming a toroid around the opening in most, though not all open ghosts. In a variable but substantial fraction of the ghosts, the initial curling was followed by a protrusive buckling of the membrane area opposite the hole leading to rapid and complete eversion of the ghost membrane, forming inside-out ghosts (IOGs) [25]. Analysis of the dynamic morphology of spontaneously vesiculating membranes in electron microscopic serial sections showed that the curls were the main IOVforming factory, both in IOGs and in the right-side-out ghosts (ROGs), those which had not buckled and everted. We shall refer to the curling-buckling-eversion-vesiculation sequence of IOGs as the CBEV sequence [23]. This particular vesiculation modality has recently acquired distinct significance in malaria-infected RBCs and is briefly described next.

Using high-speed videomicroscopy and epifluorescence, Abkarian and colleagues $[1,8]$ studied the process of merozoite egress from RBCs infected with the malaria parasite Plasmodium falciparum in culture conditions. They discovered that host-cell rupture and merozoite release at the end of the asexual reproduction cycle of the parasite occurred with the host cell membrane curling outwards around the rupture hole, forming a toroid, and then sequentially buckling, everting and vesiculating, confirming the post-egress vesiculated condition of the host cell membrane $[14,16,17]$. Buckling was shown to aid merozoite ejection and dispersal. Eversion, by removing any residual containment to dispersal, may play an important role in vivo, where egress occurs with the infected cells adhered to endothelial cells in the microvasculature $[1,23]$. Vesiculation during egress occurs extremely rapidly; the final appearance of the residual host cell membrane is that of a linked bunch of vesicles [17]. The analogies between the CBEV sequences during merozoite egress and during spontaneous vesiculation of IOGs strongly suggest common mechanisms. However, the experimental conditions and kinetics are markedly different, CBEV during egress being completed in iso-osmotic culture media within about $400 \mathrm{~ms}$ at $37^{\circ} \mathrm{C}[1]$. These findings opened a new perspective on the study of the CBEV sequence, from a process of biophysical interest in the experimental generation of inside-out plasma membrane vesicles to a process of much wider and profound biological and medical relevance, rendered amenable to study on the experimental model of the spontaneous vesiculation process.

A molecular mechanism was proposed to explain the three major features of the spontaneous vesiculation process [25]: the dynamic shaping of the emerging vesicles driven by the particular geometry of cytoskeletal breakdown, the formation of free membrane edges and the extensive membrane fusion leading to vesicular sealing. The membrane motions responsible for curling, vesicular shaping and the CBEV sequence of IOGs were attributed to the pattern of cytoskeletal disassembly and breakdown, whereas the fusion events were attributed to interactions between integral membrane proteins lining the extensive open membrane edges during intermediate vesiculation stages, acting like membrane zips.

Independent observations suggested the existence of a direct link between the modality of cytoskeletal breakdown and membrane motions during spontaneous vesiculation [20]. Spectrin (bands 1 and 2) and actin (band 7) [11, 41, 42], which make up about $75 \%$ of the cytoskeletal proteins, dissociate from the membrane in conditions of low ionic strength and $\mathrm{pH}$ similar to those in which spontaneous vesiculation occurs, and inside-out vesicles from red cell membranes prepared by different procedures were found to be depleted in spectrin and actin; the surface density of fibrillar projections from the inner membrane face, representing mostly spectrin strands $[25,30]$, was found to be much reduced in spontaneously formed vesicles relative to ghosts and intermediate vesiculation forms. These observations documented before-after conditions but provided no information about the dynamics and time-course of spectrin-actin losses during the intermediate stages of the spontaneous vesiculation process. If the membrane motions during spontaneous vesiculation result from the peculiar dissociation pattern of the spectrin cortex, it is necessary for spectrin to remain retained for as long as movement is detected. The observed depletion of spectrin-actin in the formed vesicles must therefore represent a late, terminal loss rather than the result of gradual dissociation. The work reported here focusses on the time correlation between morphology and spectrin loss and on the macroscopic deformability properties of a spectrin-free red cell membrane.

\section{Methods}

The experimental protocol for the spontaneous formation of IOVs is illustrated in Fig. 1. Briefly, fresh venous blood was 
obtained from healthy donors after informed consent, using heparinized syringes. Red blood cells were washed three times with 10 vol of a solution containing (in $\mathrm{mM}$ ) $\mathrm{NaCl} 142, \mathrm{KCl} 3$, HEPES-Na (pH 7.5) 10 and EGTA or EDTA 0.1, to chelate extracellular divalent cations. Residual plasma, buffy coat and topmost cell layer were removed after each wash. For vesiculation, the washed red cells were lysed in 50-100 vol of icecold solution "L" (in $\mathrm{mM}$ ): HEPES-Na ( $\mathrm{pH} 7.5$ ) 2.5 and EGTA 0.1 . The lysed cells were immediately spun at $15,000 \times g$ for $15-20 \mathrm{~min}$ at $0-5^{\circ} \mathrm{C}$, forming a pink pellet on top of a tiny dark button at the bottom of the centrifuged tube. The supernatant was discarded, and the pink ghost pellet was gently transferred to a new tube avoiding any contact and mixing with the dark adherent button at the bottom of the tube. This button contains residual blood cell contaminants rich in proteases capable of drastically altering the electrophoretic patterns studied here if retained, or imperfectly removed [43]. Conservation of the sodium dodecyl sulphate (SDS)-gel electrophoretic pattern of red cell membrane proteins in the current controls, even after prolonged incubations (see Fig. 5), documents the effectiveness of contaminant protease removal. The transferred ghost pellet was resuspended in ice-cold solution $\mathrm{L}$ at an equivalent hematocrit (relative to the original volume of cells) of $50-100 \%$ and the suspension preincubated in the ice-bath for 30-60 min to optimize synchronized vesiculation in the ghost population when subsequently incubated at $37^{\circ} \mathrm{C}$. Vesiculation was initiated by transferring the suspension to a water bath at $37{ }^{\circ} \mathrm{C}$. Duplicate $0.1-\mathrm{ml}$ samples of the suspension were taken before and after this transfer at the time intervals indicated in the figures and diluted ten-fold into microfuge tubes containing $0.9 \mathrm{~mL}$ of solution L with $0.1-0.5 \mathrm{mM} \mathrm{MgCl}_{2}$ to halt further vesiculation [11]. Phase contrast and Nomarski observations and photomicroscopy (Zeiss Photomicroscope III) were performed on these unfixed samples using $\times 100$ oil immersion objective lenses and temperature-controlled slides.

Samples for SDS-polyacrylamide gradient gel electrophoresis were processed as originally described by Fairbanks and collaborators [11]. The gels were fixed and stained with Coomassie Brilliant Blue. The Mg-arrested samples were ice-cooled and centrifuged at $12,000 \times \mathrm{g}$ for $30 \mathrm{~min}$ in a temperature-controlled microfuge at $4{ }^{\circ} \mathrm{C}$ (Eppendorff Centrifuge 5402). The membrane pellets and supernatants were separated and independently processed for electrophoresis.
Fig. 1 Sequential steps in the experimental protocol for the spontaneous formation of insideout vesicles. Pre-incubation of the ghost membranes at $0{ }^{\circ} \mathrm{C}$ improves vesiculation synchrony in the ghost population during subsequent incubation at $37^{\circ} \mathrm{C}$ (see text). The figure at the bottom illustrates a sequence of Nomarski views $(\times 1,000)$ taken from samples at 1, 2, 3, 4, 6 and $30 \mathrm{~min}$ of incubation at $37^{\circ} \mathrm{C}$. After the spin (step 3 from top), the supernatant was discarded and the pink ghost pellet was gently transferred to a new tube for subsequent resuspension in $\mathrm{L}$ (step 4 from top), taking care to prevent any contact and mixing with the dark adherent button at the bottom of the centrifuged tube (see "Methods")
Fresh human erythrocytes washed and packed ( 80\% Hct)

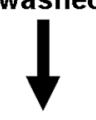

Lyse at $0-4^{\circ} \mathrm{C}$ in $50-100$ volumes of solution $\mathrm{L}$ : HEPES-Na (pH 7.5-7.6), $2 \mathrm{mM}$ Na-EDTA or Na-EGTA, $0.1 \mathrm{mM}$

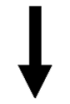

Spin at $15000 \mathrm{~g}$ for $20 \mathrm{~min}$ at $4^{\circ} \mathrm{C}$

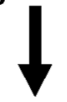

Resuspend ghosts in $L$ at $\sim \mathbf{5 0 - 1 0 0 \%}$ equivalent $\mathrm{Hct}$

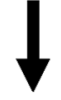

Pre-incubate at $\sim 0^{\circ} \mathrm{C}$ for $30-60 \mathrm{~min}$

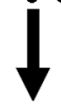

Incubate at $37^{\circ} \mathrm{C}$ (min)

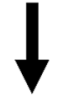

Samples in $\mathrm{L}+$ 0.1-0.5 $\mathrm{mM} \mathrm{MgCl} 2$

for morphology and membrane proteins (min; Nomarski x 1000)
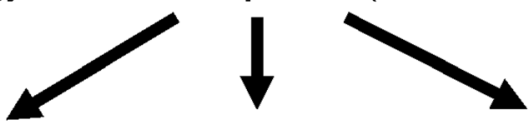

0

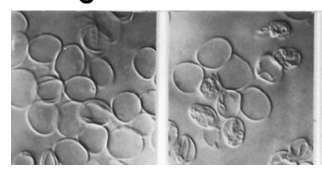

3
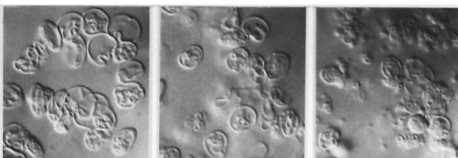

6

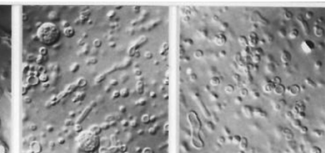


The supernatant sample was used to identify the membrane proteins that became fully detached and lost to the medium during the vesiculation process.

\section{Results}

Throughout this investigation, we have made extensive use of four empirical observations that will be documented in more detail in the following sections: (i) Pre-incubation of the lysed RBCs at $0{ }^{\circ} \mathrm{C}$ for about $30-60$ min immediately after lysis was found to increase the level of synchronization of the vesiculation process among different ghosts relative to non-preincubated samples, important for studying the correlation between morphology and membrane protein changes in multi-cell samples of spontaneously vesiculating ghost. The lysis conditions in which IOVs are spontaneously formed (low-ionic strength, divalent cation-free hypotonic media) were shown to prevent sealing of the lytic hole in all ROGs. This opening in all ROGs, as well as the everted condition of the IOGs, ensure free access of the ice-cold lysis medium to the inner membrane surfaces during the pre-incubation period. Synchronization is thus probably the result of a slow coordinated rearrangement of the cytoskeleton to a similar starting configuration in most ghosts; (ii) addition of small concentrations of $\mathrm{MgCl}_{2}(0.1-0.5 \mathrm{mM})$ to suspensions of vesiculating ghosts instantly and reversibly arrests the vesiculation process. $\mathrm{Mg}$ arrest could therefore be used to freeze vesiculating samples in time thus enabling study of time-dependent changes on stabilized, unfixed samples. Other divalent cations $\left(\mathrm{Ca}^{2+}, \mathrm{Co}^{2+}\right)$ also cause instant vesiculation arrest, but reversibility was only observed after $\mathrm{Mg}^{2+}$ washout and resuspension of the membranes in the divalent cation-free lysis medium. Evidence will be provided that suggests that the vesiculation-arresting effects of divalent cations result from spectrin cross-linking; (iii) to investigate the changes in membrane proteins during $\mathrm{IO}$ vesiculation, it was found convenient to retain a trace haemoglobin $(\mathrm{Hb})$ concentration in the medium $(5-10 \mu \mathrm{M})$ to be used as an indicator of vesicular sealing by its increased membrane association within a newly sealed membrane compartment during sample elution; and (iv) a relatively minor increase in the ionic strength of the lysis and vesiculation medium had no significant effect on the rate of cytoskeletal breakdown, but it dramatically reduced the extent of spontaneous vesiculation leaving a preparation in which most ghosts had the appearance of giant liposomes. This allowed us to explore the physical behaviour of cytoskeleton-free membranes under convective currents.

In the experiment of Fig. 2, red cells were lysed and preincubated in solution $\mathrm{L}$ in the cold as per the protocol in Fig. 1 and subsequently re-suspended and incubated at $37{ }^{\circ} \mathrm{C}$ in medium $\mathrm{L}$ with added $\mathrm{NaCl} 10 \mathrm{mM}$. Mg-arrested samples were placed between slide and coverslip and recorded under phase contrast observation. Spontaneous vesiculation was much reduced under these conditions, but ghost appearance changed dramatically as cytoskeletal proteins detached, from an initial stiff, folded body (Fig. 2a) to that of a soft, inflated balloon, easily elongated along minor convective currents (Fig. 2b). Figure 2c, from a 30-min sample, illustrates the effect of gently tapping the coverslip on the appearance of such ghosts under the convective currents generated by this manoeuvre. It can be seen that the membranes deformed into extended tubular structures as would be expected from a twodimensional liquid devoid of intrinsic shape controls. This simple manoeuvre illustrates the inability of cytoskeleton-free membranes to generate shape changes in the absence of external forces. The spontaneous membrane motions during IO vesiculation must therefore be driven by localized configuration changes within the cortical cytoskeletal mesh as it breaks down. Because these motions take place in the total absence of energy sources other than thermal, they are entropic and must result from potential-to-kinetic energy conversions within the disassembling cytoskeleton, the modality of breakdown of the cortical cytoskeletal mesh shaping the curls and emerging vesicular forms [20]. As argued in the "Introduction", a necessary condition for this interpretation is that spectrin and actin, the main cytoskeletal mesh components, should be retained for as long as spontaneous motions persist, and we report next the results of experiments designed to test this condition.

Membrane protein changes were followed on pellets and supernatants of vesiculating samples by SDS-polyacrylamide gel electrophoresis (see "Methods"). Figure 3 illustrates the initial $(\mathrm{G})$ and final $(\mathrm{V})$ conditions of membrane proteins in spontaneously vesiculating ghost preparations. The main components of the cytoskeletal mesh are spectrin (bands 1 and 2) and actin (band 5). The spectrin subunits $\alpha$ and $\beta$ have MWs 220 and $240 \mathrm{kDa}$, respectively, and the MW of the actin monomers is $43 \mathrm{kDa}$. The band which corresponds to globin from haemoglobin monomers, the SDS denatured form of the solubilised haemoglobin tetramer, has a MW of about $16 \mathrm{kDa}$. The results (Fig. 3) document a dramatic loss of spectrin and actin in spontaneously formed IOVs $(\mathrm{V})$ relative to prevesiculation ghost membranes $(\mathrm{G})$, a pattern similar to that recorded for IO vesicles prepared by the Steck-Kant method $[11,29]$. The small and variable residual amount of spectrinactin associated with vesiculated membrane pellets can be attributed to asynchronies and heterogeneities in vesiculation patterns: partially vesiculated ghosts, incomplete detachment of spectrin strands visible as isolated residual fibrillar projections in electron microscopic images $[20,25,30]$ and detached cytoskeletal proteins retained within sealed vesicles. The reduction in spectrin and actin contrasts with the dramatic increase in membrane-associated haemoglobin (Fig. 3, V relative to $G)$. To establish whether this haemoglobin was bound to or trapped within the vesicles, the vesiculated membranes were exposed to two consecutive freezing-thawing 

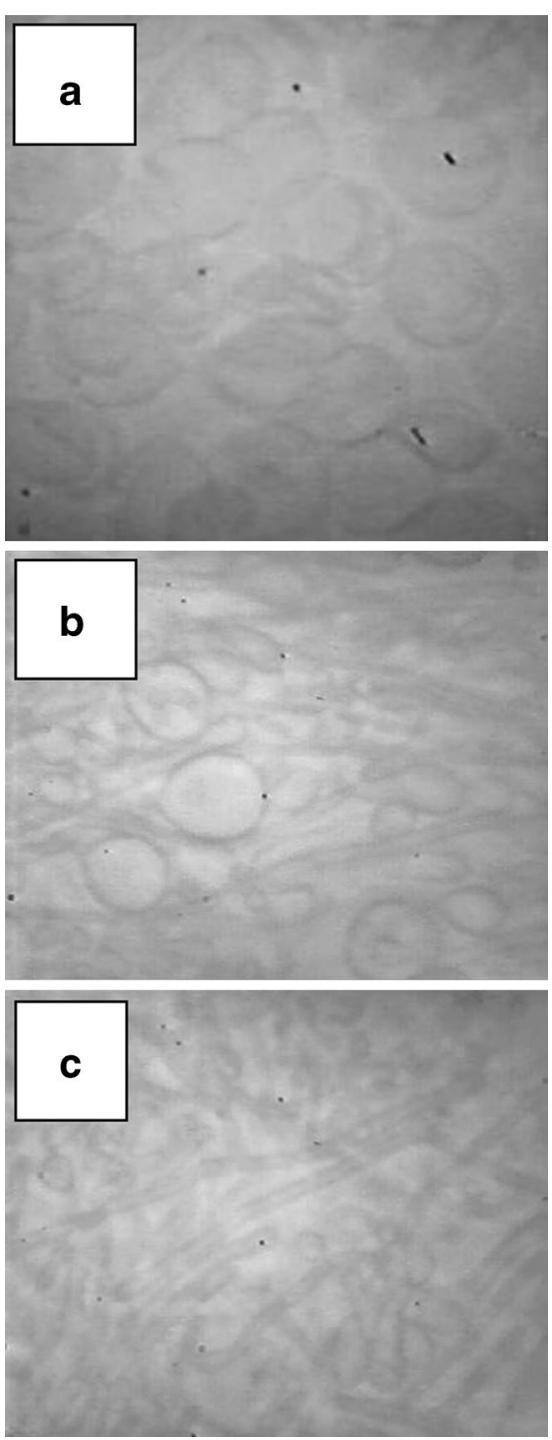

Fig. 2 Deformability of cytoskeleton-free red cell membranes under convective currents. a Ghosts lysed in solution L and incubated at $37^{\circ} \mathrm{C}$ in solution $\mathrm{L}$ with added $10 \mathrm{mM} \mathrm{NaCl}$ for $30 \mathrm{~min}$. These conditions largely de-couple spectrin-actin loss from spontaneous vesiculation leaving cytoskeleton-depleted, partially vesiculated ghosts. b, c Effects of mild (b) and strong (c) tapping of the coverslip on ghost membrane shape changes. The convective currents generated by the tapping were left to subside before the pictures were taken. They illustrate the non-reversibility of the membrane deformations generated by external forces on cytoskeleton-free membranes

cycles to disrupt permeability barriers. Freezing-thawing prior to SDS-gel electrophoresis fully removed all $\mathrm{Hb}-$ membrane association in the vesiculated samples (not shown), indicating that the observed membrane-associated $\mathrm{Hb}$ had been trapped within sealed vesicles rendered permeable by freezing and thawing.

The time-dependent changes in $\mathrm{RBC}$ membrane proteins throughout the spontaneous IO vesiculation process were followed in parallel with morphological changes under Nomarski optics observation (Fig. 4). During the first 5 min,

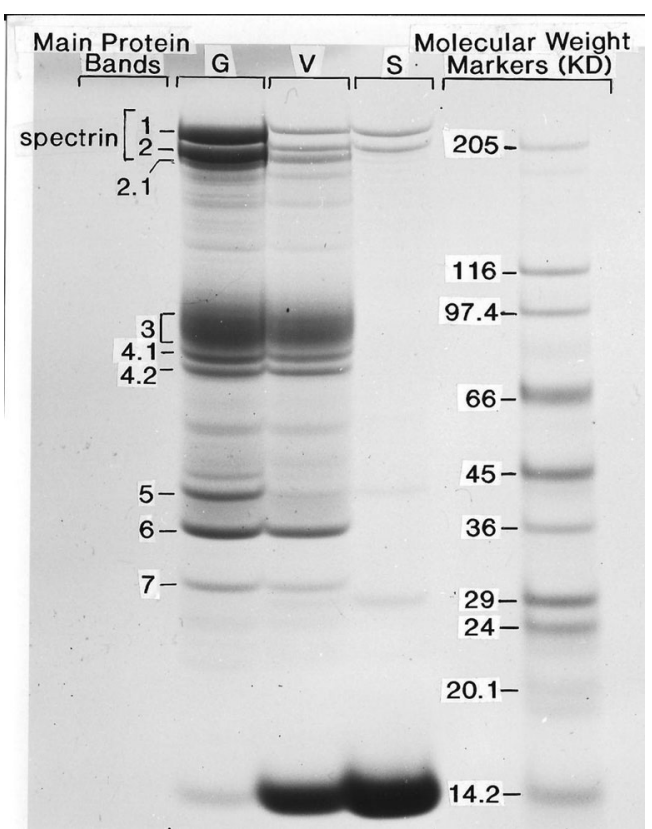

Fig. 3 Membrane protein changes during spontaneous inside-out vesiculation. The first there columns (left to right) show representative SDSgel electrophoretic patterns of pre-vesiculation ghosts $(G)$, post-vesiculation IOVs $(V)$ and post-vesiculation supernatants $(S)$. The rightmost column indicates distances travelled by molecular weight markers on the same gel. The band numbers on the left-most column follow the familiar nomenclature established in the pioneering papers of Fairbanks, Steck and collaborators $[11,42]$. The most relevant electrophoretic components in the context of the present work are bands 1 and 2 (spectrin), actin (band 7) and monomeric haemoglobin (16 kDa, next to the 14.2$\mathrm{kDa} \mathrm{MW}$ marker in this figure; the bottom band in all membrane protein columns). Because spectrin and actin form the bulk of the cytoskeletal mesh of the red blood cell cortical cytoskeleton, the focus of this investigation is on their time-dependent changes during spontaneous vesiculation. Haemoglobin traces are used to signal the time of vesicular sealing, as explained in "Results". Although the same volume of sample was deposited on the gels for $\mathrm{G}$ and $\mathrm{V}$ samples, $\mathrm{V}$ samples carried less total protein than $\mathrm{G}$ samples because of losses to supernatant. The supernatant column $(S)$ identifies the membrane proteins that were fully detached and released to the incubation medium during vesiculation despite their highly diluted state relative to their density in the membrane samples. The monomeric globin band circa $16 \mathrm{kDa}$ can be clearly seen in the $\mathrm{V}$ and $\mathrm{S}$ samples but not in the open state G samples

we see profound morphological changes along previously described patterns [25] with hardly any detectable change in membrane proteins. The release of spectrin and actin to the supernatant becomes detectable only after the 5 -min sample in this series. Spectrin detachment from the membranes to the supernatant is progressively evident in the 6- and 8-min samples, and the weaker actin band is clearly detectable in the 8min supernatant sample. Between the fifth and sixth minute, there is an abrupt increase in the membrane-associated monomeric $\mathrm{Hb}$ band, in parallel with late formation of vesicles. These results clearly show that the main cytoskeletal mesh components, spectrin and actin, remain associated with the cell membrane during the dynamic morphological changes leading to vesiculation and that they only become fully 


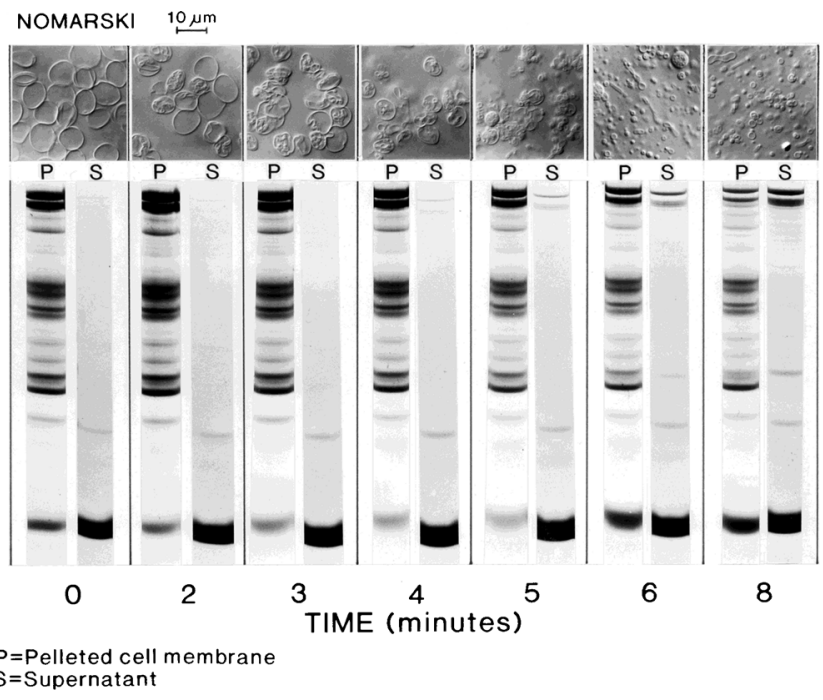

Fig. 4 Time relations between morphological and membrane protein changes during spontaneous inside-out vesiculation of red cell membranes. Red cells were lysed and resuspended at $50 \%$ equivalent haematocrit in $\mathrm{L}$ at $0{ }^{\circ} \mathrm{C}$. The suspension was kept at $0{ }^{\circ} \mathrm{C}$ in an ice-bath. A 5- $\mu \mathrm{L}$ sample was placed between slide and coverslip on a temperaturecontrolled stage, initially set at $4{ }^{\circ} \mathrm{C}$, of a Zeiss photomicroscope under Nomarski $(\times 1,000)$ observation. Vesiculation was initiated $(t=0)$ both in the suspension and slide by transfer to a water bath at $37^{\circ} \mathrm{C}$ and by rising the stage temperature to $37^{\circ} \mathrm{C}$, respectively. Suspension samples for membrane (left of paired columns) and supernatant (right of paired columns) proteins were taken at the indicated times and processed as described in "Methods". Nomarski photo-images were taken at the indicated times and are shown in the figure lined up above the time-corresponding membrane protein columns. Note that the reduction of spectrin from pellets (bands 1 and 2, Fig 3) and the appearance of spectrin in supernatants become detectable at about $5 \mathrm{~min}$, just before large-scale vesicular sealing as indicated by haemoglobin trapping (bottom band). Allowing for imperfect synchronization, the results suggest large-scale spectrin retention during the early dynamic stages of the spontaneous vesiculation process

dissociated at the final vesicle sealing stage, when $\mathrm{Hb}$ becomes trapped within sealed vesicles.

Figure 5 shows the results of an experiment similar to that of Fig. 4 in which the changes in membrane and supernatant proteins during spontaneous vesiculation were followed at minute intervals for $8 \mathrm{~min}$ to a final 20 -min sample. In this experiment, in addition, duplicate $\mathrm{Mg}$-arrested samples were incubated for a further $1 \mathrm{~h}$ at $37^{\circ} \mathrm{C}$ before SDS-gel processing to investigate whether the observed $\mathrm{Mg}$ arrest of vesiculation also prevented spectrin-actin release, as expected if the reversible arrest of all spontaneous dynamic motion was the result of a freeze in cytoskeletal configuration at the instant of interaction with the divalent cation.

The results (Fig. 5, left panels) confirm with greater detail than in Fig. 4 the time-course of spectrin-actin retention and loss and of $\mathrm{Hb}$ trapping within sealing vesicles. Full retention of spectrin and actin during the first 4 to $5 \mathrm{~min}$, that is, during the most dynamic stage of the vesiculation process, is particularly noteworthy because it supports the role of the cytoskeletal mesh as the dynamic drive of spontaneous vesiculation.
Post-incubation in the Mg-arrest medium (Fig. 5, right panels) reveals a similar pattern of spectrin-actin retention-loss to that of the non-post-incubated samples, the pattern expected from a stabilizing role of $\mathrm{Mg}$ ions on the spectrin-actin configuration of the membranes at the instant of Mg exposure. Reversibility of the $\mathrm{Mg}$ effect was demonstrated by the continuity of the vesiculation process after washing $\mathrm{Mg}$ away and further incubation of the membranes in the lysis medium (not shown). Together with the results in Figs. 4 and 5, the implication is that the configuration of the cytoskeletal mesh at arrest fully recovers its pre-arrest condition after $\mathrm{Mg}$ removal.

We consider next whether or not spectrin-actin detachment during inside-out vesiculation occurs uniformly over the whole membrane area, a question amenable to test by a procedure based on earlier work by Lange and collaborators [22]. Naked membrane patches devoid of spectrin reticulum, if formed during spontaneous inside-out vesiculation, could be revealed by exposing the membranes to intense shearing forces. As shown in Fig. 2, naked membranes deform markedly even under relatively mild convective currents. Exposed to intense shear, they fragment and reseal into tiny vesicles. The volume of membrane pellet $(V)$ would be expected to be reduced as the mean equivalent radius $(r)$ of the emerging vesicles decreases. At constant membrane area, assuming spherical ghost-to-vesicle conversions, $V \sim(A / 3) r$. We measured the volume of membrane pellets in $\mathrm{Mg}$-arrested samples throughout spontaneous inside-out vesiculation, in paired samples, with and without vortexing for $30 \mathrm{~s}$. Volume reductions in unvortexed samples reflect mean radius reductions resulting from spontaneous vesiculation; in vortexed samples, they expose additional shear-induced vesiculation on naked membrane patches. The results in Fig. 6 show that the major extent of volume reduction is due to spontaneous vesiculation (74 to $88 \%$ in the four experiments of this series), with a significant and gradually increasing availability of naked membranes patches to additional fragmentation and vesiculation (Fig. 6, triangles).

\section{Discussion}

The results presented here show that, within the bounds of attainable synchronization of spontaneous vesiculation in the ghost population, extensive spectrin-actin detachment and release to supernatant occurs only at the stage when haemoglobin retention exposes vesicular sealing (Figs. 4 and 5). The bulk of the spectrin-actin loss is therefore a terminal event, concurrent with terminal vesicular sealing (Figs. 3, 4 and 5). Further incubation of Mg-arrested samples taken throughout vesiculation does not elicit additional membrane protein loss or altered globin association relative to samples processed immediately (Fig. 5), consistent with a reversible 


\section{Membrane proteins}

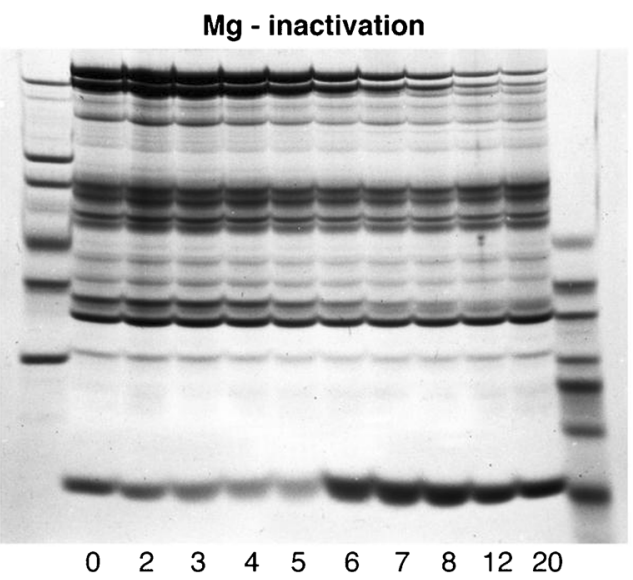

Mg - inactivation/incubation

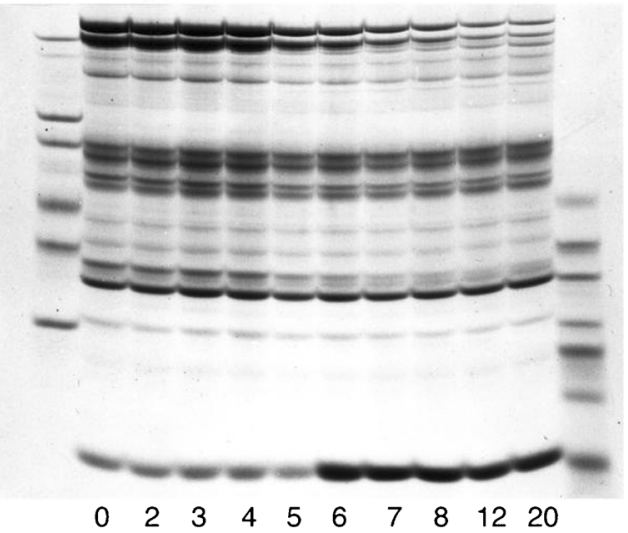

Membrane proteins lost to medium
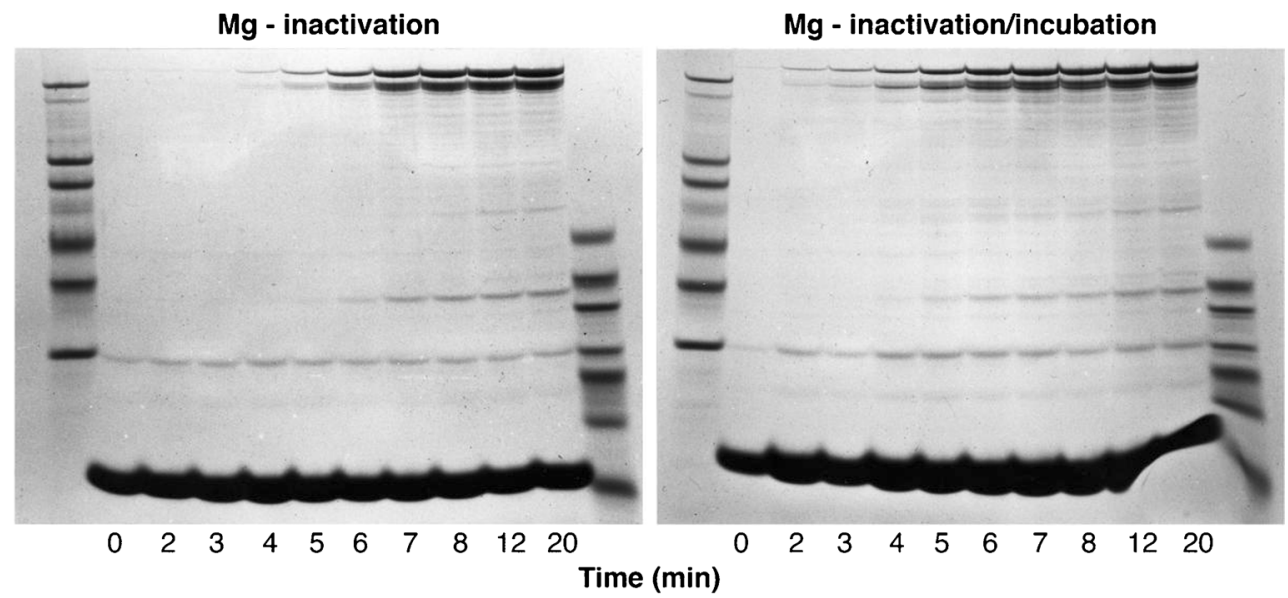

Fig. 5 Effect of magnesium ions on the time-dependent patterns of membrane protein changes during spontaneous vesiculation. The experimental protocol was similar to that described in Fig 4. Samples for membrane and supernatant proteins shown on the left panels were taken before $(t=0)$ and after switching the temperature of the ghost suspension from 0 to $37^{\circ} \mathrm{C}$, at the times indicated under each column (in min). Left panels show the patterns obtained from $\mathrm{Mg}$-inactivated samples immediately processed for SDS-gel electrophoresis, as reported in "Methods" (Mg inactivation). Right panels show the patterns recovered from duplicate $\mathrm{Mg}$-inactivated samples from each of the originally timed samples after $1 \mathrm{~h}$ of incubation at $37^{\circ} \mathrm{C}$ before processing for SDS-gel electrophoresis (Mg inactivation/incubation). Extreme left and right columns in

cross-linking effect of $\mathrm{Mg}$ ions stabilizing the configuration of the spectrin-actin mesh at the time of $\mathrm{Mg}$ exposure.

Electron microscopic images of vesiculating membranes from both right-side out and inside-out ghosts showed timedependent changes in the appearance of the fibrillar projections lining the inner membrane surface, the morphological correlate of the spectrin-actin mesh [25]. The fibrillar projections changed from an initial uniform distribution to a progressively flaky and patchy appearance representing higherorder spectrin or spectrin-actin aggregates. Some of these aggregates could be seen terminally as fully detached fibres each of the four panels are molecular weight standards. Top panels show membrane proteins; corresponding bottom panels show bands of proteins lost to the supernatant. Notwithstanding imperfect synchronization, it is clear that there is no large-scale spectrin loss (bands 1 and 2, see Fig 3) from membranes to supernatants during the first 4 to $5 \mathrm{~min}$, the most dynamic stages of the spontaneous vesiculation process. Large-scale spectrin loss occurs concurrently with haemoglobin retention in the membrane protein gels (6-min sample) reflecting vesicular sealing. The similitude of the two membrane protein gels (top panels), particularly for the samples taken during the first 4 to $5 \mathrm{~min}$ of incubation, supports the view that vesiculation arrest by $\mathrm{Mg}^{2+}$ results from prevention of spectrin detachment, probably through spectrin cross-linking

retained within sealed vesicles. The present results (Fig. 6) document progressive formation of naked membrane patches during vesiculation, a correlate to the changing fibrillar morphology. Our data also provides approximate estimates of the relative contributions of active (dependent on spectrin cortex dynamics) and passive (shear-induced on naked membrane patches) components to reductions in mean vesicular radius during spontaneous vesiculation, of about 80 and $20 \%$, respectively.

In the original molecular model of the spontaneous vesiculation process [25], retention of a spectrin cortex undergoing 


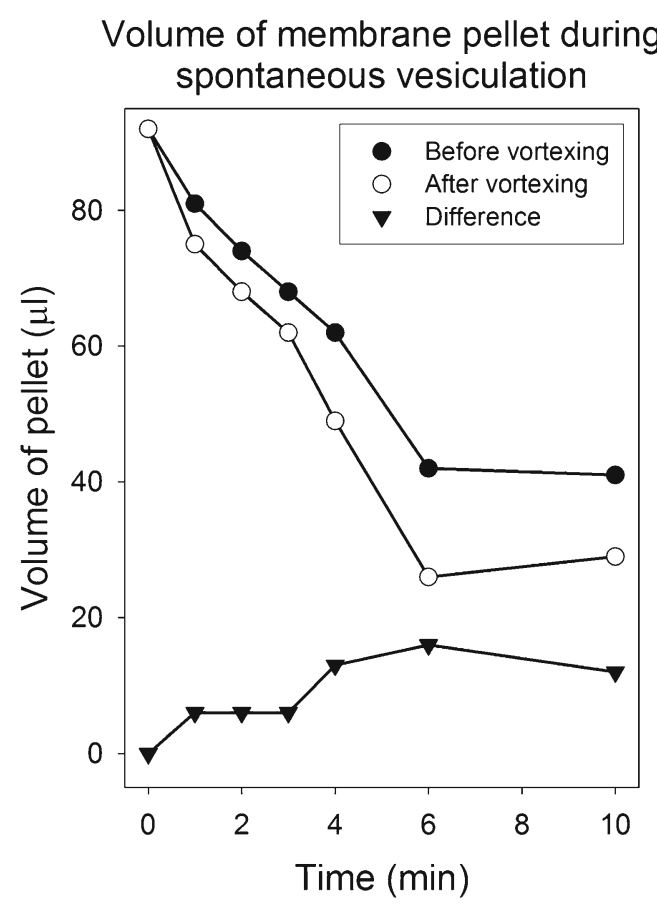

Fig. 6 Shear-induced changes in the volume of membrane pellets during spontaneous vesiculation. Red cells were lysed and resuspended at $50 \%$ equivalent haematocrit in $\mathrm{L}$ at $0{ }^{\circ} \mathrm{C}$. After $30 \mathrm{~min}$ pre-incubation in the ice-bath, vesiculation was initiated $(t=0)$ by transfer to a water bath at $37^{\circ} \mathrm{C}$. Paired Mg-arrested samples were taken at the indicated times. One sample of each pair was vigorously vortexed for $30 \mathrm{~s}$, and both samples were then centrifuged at $12,000 \times \mathrm{g}$ for $30 \mathrm{~min}$ at $4{ }^{\circ} \mathrm{C}$, conditions that left no detectable membrane protein presence in the supernatants. The volume of the membrane pellets was estimated from the height of the pellet column on magnified photo records of the microfuge tubes which had been volumetrically calibrated with coloured fluids (the coefficient of variation of the volume estimates was $5.8 \%$ ). Results are representative of four similar experiments

profound structural changes during the dynamic stages of the vesiculation process was considered necessary for vesicular modelling. The current results confirm large-scale spectrin retention during the membrane dynamic shape changes and large-scale detachment at the terminal vesiculated stage (Figs. 4 and 5). However, the mechanisms by which the structural changes in the spectrin cortex control membrane dynamics remain to be elucidated. Three different mechanisms were proposed in the recent literature to account for membrane dynamics and shape control: (i) localized release of spectrin constraints on the sign and dynamics of the membrane curvature [18, 20, 37], (ii) elastic energy release [1, 8] and (iii) spectrin-oligomerization-driven shape changes [35]. The first two are theoretical models focussed on curling and on egress-associated membrane dynamics in conditions of altered spectrin cortex structure. The third is experimentally based and concerns shape control in intact red blood cells. Nans and collaborators [35] applied cryo-electron tomography to study the topology of the cytoskeletal mesh in intact, unfixed mouse red cells, frozen in physiological buffer. From analysis of the contour length of the spectrin filaments connecting junctional complexes, relative to the fully extended length of the spectrin heterotetramer, they concluded that higher-order oligomers, mostly hexamers and octamers, were prevalent in the mesh network and suggested that spectrin oligomerization dynamics may be central to cell shape control. It is therefore a plausible option that even in the unphysiological conditions in which spontaneous vesiculation occurs, local spectrin oligomerization dynamics, freed from divalent cation stabilizing influences, helps sculpt some of the complex shape transformations of the vesiculating membranes for as long as actin nodal links persist.

A comparative analysis of the CBEV sequences in IOGs and during merozoite egress provides some insights on the likelihood of these alternative mechanisms. In both hypoosmotic lysis in low-ionic strength, divalent cation-free media and pre-egress host-cell rupture in iso-osmotic, plasma-like media, a large opening is formed in the red cell membrane. In IOGs and after pre-egress rupture, as soon as the hole is formed, the membrane rapidly curls, buckles and everts (the CBE response). But whereas in IOGs curling, buckling and eversion constitute the response of an initially normalconfigured cytoskeleton, in the infected cells the CBE response occurs with an extensively remodelled cytoskeleton resulting from pre-egress protease activity $[6,9,14,15,33$, 34]. IOGs would never form in the presence of divalent cations or in isotonic, high ionic strength media. CBE prevention by divalent cations in IOGs probably results from spectrin cross-linking, consistent with the results in Fig. 5. On the other hand, egress membranes undergo $\mathrm{CBE}$ after rupture in plasma-like media with relatively high $\mathrm{Ca}^{2+}$ and $\mathrm{Mg}^{2+}$ concentrations. One possible interpretation is that in infected RBCs, pre-egress cytoskeletal remodelling removes the capacity of divalent cations to cross-link spectrin, as if the molecular distances between spectrin strands in the disrupted cytoskeletal mesh became no longer bridgeable by crosslinking action. Indirectly, this line of argument would tend to support constrain release mechanisms for CBE in IOGs and egress because it is hard to envisage similar spectrin oligomerization responses from such different initial cytoskeletal constitutions.

IOGs form instantly upon lysis at $0{ }^{\circ} \mathrm{C}$ [25]. The timecourses of CBE in IOGs at $0{ }^{\circ} \mathrm{C}$ and of CBEV on egress at $37^{\circ} \mathrm{C}$ [1] appear similarly rapid, both completed in a fraction of a second. On the other hand, terminal vesiculation from IOGs takes 5-8 $\mathrm{min}$ at $37^{\circ} \mathrm{C}$ (Figs. 1, 4 and 5), much longer than the $\sim 400 \mathrm{~ms}$ for post-egress vesiculation. The kinetic differences between the two CBEV sequences are thus essentially confined to the $E-V$ interval. But the $V$ differences between the two processes are more profound. When fluorescent phalloidin A was present in the culture medium during egress, the vesiculated residual red cell membranes appeared strongly labelled with the fluorescent dye indicating substantial retention of the pre-egress cytoskeleton in the post-egress 
vesiculated membrane [14]. On the other hand, in lysisgenerated IOVs, whether IOGs or ROGs, the spectrin cortex has dissociated (Figs. 4 and 5). The vesicles in the post-egress membrane remain aggregated, like bunch of grapes [14], whereas IOVs are mostly free (Figs. 1 and 4), reflecting the persistence (in egress membranes) and loss (in IOVs) of structural linking elements. The few high-speed videorecords of egress in which vesiculation can be discerned [1, 10] show vesiculated membranes arising from the everting toroid, as was shown for lysis-formed IOVs, suggesting similar cutting-splicing processes and topologies in the residual vesiculated state, although the inside-out sidedness of the egress-formed vesiculated membrane residue is yet to be established. Whether a common molecular mechanism may account for such profound structural and formation-rate differences between IOVs and post-egress vesicles remains an open question prompting further research.

Acknowledgments The authors wish to thank The Wellcome Trust (UK) for research funds and to Austin Hockaday for technical help.

Ethical standards The authors declare that the experiments comply with the current laws of the country in which they were performed.

Conflict of interest The authors declare that they have no conflict of interest.

Open Access This article is distributed under the terms of the Creative Commons Attribution License which permits any use, distribution, and reproduction in any medium, provided the original author(s) and the source are credited.

\section{References}

1. Abkarian M, Massiera G, Berry L, Roques M, Braun-Breton C (2011) A novel mechanism for egress of malarial parasites from red blood cells. Blood 117:4118-4124

2. Alvarez J, Garcia-Sancho J, Herreros B (1988) All or none cell responses of $\mathrm{Ca} 2+$-dependent $\mathrm{K}$ channels elicited by calcium or lead in human red cells can be explained by heterogeneity of agonist distribution. J Membr Biol 104:129-138

3. Begenisich T, Nakamoto T, Ovitt CE, Nehrke K, Brugnara C, Alper SL et al (2004) Physiological roles of the intermediate conductance, Ca2+-activated potassium channel Kcnn4. J Biol Chem 279:4768147687

4. Bennett V, Branton D (1977) Selective association of spectrin with the cytoplasmic surface of human erythrocyte plasma membranes. J Biol Chem 252:2753-2763

5. Bennett V, Stenbuck PJ (1979) The membrane attachment for spectrin is associated with band 3 in human erythrocyte membranes. Nature 280:468-473

6. Blackman MJ (2008) Malarial proteases and host cell egress: an 'emerging' cascade. Cell Microbiol 10:1925-1934

7. Branton D, Cohen CM, Tyler J (1981) Interaction of cytoskeletal proteins on the human erythrocyte membrane. Cell 24:24-32

8. Callan-Jones A, Albarran Arriagada OE, Massiera G, Lorman V, Abkarian M (2012) Red blood cell membrane dynamics during malaria parasite egress. Biophys J 103:2475-2483
9. Chandramohanadas R, Davis PH, Beiting DP, Harbut MB, Darling C, Velmourougane G et al (2009) Apicomplexan parasites co-opt host calpains to facilitate their escape from infected cells. Science 324:794-797

10. Crick AJ, Tiffert T, Shah SM, Kotar J, Lew VL, Cicuta P (2013) An automated live imaging platform for studying merozoite egressinvasion in malaria cultures. Biophys J 104:997-1005

11. Fairbanks G, Steck TL, Wallach DFH (1971) Electrophoretic analysis of the major polypeptides of the human erythrocyte membrane. Biochemistry 10:2606-2617

12. Garcia-Sancho J, Sanchez A, Herreros B (1982) All-or-none response of the $\mathrm{Ca} 2+-$ dependent $\mathrm{K}+$ channel in inside-out vesicles. Nature 296:744-746

13. Gardos G (1958) The function of calcium in the potassium permeability of human erythrocytes. Biochim Biophys Acta 30:653-654

14. Glushakova S, Humphrey G, Leikina E, Balaban A, Miller J, Zimmerberg J (2010) New stages in the program of malaria parasite egress imaged in normal and sickle erythrocytes. Curr Biol 20:11171121

15. Glushakova S, Mazar J, Hohmann-Marriott MF, Hama E, Zimmerberg J (2009) Irreversible effect of cysteine protease inhibitors on the release of malaria parasites from infected erythrocytes. Cell Microbiol 11:95-105

16. Glushakova S, Yin D, Gartner N, Zimmerberg J (2007) Quantification of malaria parasite release from infected erythrocytes: inhibition by protein-free media. Malar J 6:61-65

17. Glushakova S, Yin D, Li T, Zimmerberg J (2005) Membrane transformation during malaria parasite release from human red blood cells. Curr Biol 15:1645-1650

18. Gov N, Cluitmans J, Sens P, Bosman GJ (2009) Cytoskeletal control of red blood cell shape: theory and practice of vesicle formation. Elsevier, Oxford, pp 95-119

19. Hoffman JF, Joiner W, Nehrke K, Potapova O, Foye K, Wickrema A (2003) The hSK4 (KCNN4) isoform is the $\mathrm{Ca}^{2+}$-activated $\mathrm{K}^{+}$channel (Gardos channel) in human red blood cells. Proc Natl Acad Sci U S A 100:7366-7371

20. Kabaso D, Shlomovitz R, Auth T, Lew VL, Gov NS (2010) Curling and local shape changes of red blood cell membranes driven by cytoskeletal reorganization. Biophys J 99:808-816

21. Kotlyar AB, Borovok N (2002) NADH oxidation and NAD+ reduction catalysed by tightly coupled inside-out vesicles from Paracoccus denitrificans. Eur J Biochem 269:4020-4024

22. Lange Y, Hadesman RA, Steck TL (1982) Role of the reticulum in the stability and shape of the isolated human erythrocyte membrane. $\mathrm{J}$ Cell Biol 92:714-721

23. Lew VL (2011) Malaria: surprising mechanism of merozoite egress revealed. Curr Biol 21:R314-R316

24. Lew VL, Ferreira HG (1978) Calcium transport and the properties of a calcium-activated potassium channel in red cell membranes. In: Kleinzeller A, Bronner F (eds) Current topics in membranes and transport, vol 10. Academic, New York, pp 217-277

25. Lew VL, Hockaday A, Freeman CJ, Bookchin RM (1988) Mechanism of spontaneous inside-out vesiculation of red cell membranes. J Cell Biol 106:1893-1901

26. Lew VL, Muallem S, Seymour CA (1982) Properties of the $\mathrm{Ca}^{2+}$ activated $\mathrm{K}^{+}$channel in one-step inside-out vesicles from human red cell membranes. Nature 296:742-744

27. Lieber MR, Steck TL (1982) A description of the holes in human erythrocyte membrane ghosts. J Biol Chem 257:11651-11659

28. Lieber MR, Steck TL (1982) Dynamics of the holes in human erythrocyte membrane ghosts. J Biol Chem 257: $11660-11666$

29. Liu SC, Fairbanks G, Palek J (1977) Spontaneous, reversible protein cross-linking in the human erythrocyte membrane. Temperature and pH dependence. Biochemistry 16:4066-4074 
30. Marchesi VT, Palade GE (1967) The localization of Mg-Na-Kactivated adenosine triphosphate on red cell ghost membranes. J Cell Biol 35:385-404

31. Marin R, Proverbio T, Proverbio F (1986) Inside-out basolateral plasma membrane vesicles from rat kidney proximal tubular cells. Biochim Biophys Acta 858:195-201

32. Mercer RW, Dunham PB (1981) Membrane-bound ATP fuels the $\mathrm{Na} / \mathrm{K}$ pump. Studies on membrane-bound glycolytic enzymes on inside-out vesicles from human red cell membranes. J Gen Physiol 78:547-568

33. Millholland MG, Chandramohanadas R, Pizzarro A, Wehr A, Shi H, Darling C et al (2011) The malaria parasite progressively dismantles the host erythrocyte cytoskeleton for efficient egress. Mol Cell Proteomics 10:M111

34. Millholland MG, Mishra S, Dupont CD, Love MS, Patel B, Shilling D et al (2013) A host GPCR signaling network required for the cytolysis of infected cells facilitates release of apicomplexan parasites. Cell Host Microbe 13:15-28

35. Nans A, Mohandas N, Stokes DL (2011) Native ultrastructure of the red cell cytoskeleton by cryo-electron tomography. Biophys J 101: 2341-2350

36. Sarkadi B, Szebeni J, Gardos G (1980) Effects of calcium on cation transport processes in inside-out red cell membrane vesicles. In: Lassen UV, Ussing HH, Wieth JO (eds) Membrane transport in erythrocytes. Relations between function and structure. Alfred Benzon symposium 14. Munksgaard, Copenhagen, pp 220-231

37. Sens P, Gov N (2007) Force balance and membrane shedding at the red-blood-cell surface. Phys Rev Lett 98:018102
38. Shotton DM (1983) The proteins of the erythrocyte membrane. In: Harris JR (ed) Electron microscopy of proteins, vol 4. Academic, London, pp 205-330

39. Shotton DM, Burke BE, Branton D (1979) The molecular structure of human erythrocyte spectrin. J Mol Biol 131:303-329

40. Shotton DM, Thompson K, Wofsy L, Branton D (1978) Appearance and distribution of surface proteins of the human erythrocyte membrane. J Cell Biol 76:512-531

41. Steck TL (1972) Cross-linking the major proteins of the isolated erythrocyte membrane. J Mol Biol 66:295-305

42. Steck TL (1974) The organization of proteins in the human red blood cell membrane. J Cell Biol 62:1-19

43. Steck TL, Kant JA (1974) Preparation of impermeable ghosts and inside-out vesicles from human erythrocyte membranes. Methods Enzymol 31:172-180

44. Steck TL, Weinstein RS, Straus JH, Wallach DFH (1970) Inside-out red cell membrane vesicles: preparation and purification. Science 168:255-257

45. Vandorpe DH, Shmukler BE, Jiang L, Lim B, Maylie J, Adelman JP et al (1998) cDNA cloning and functional characterization of the mouse $\mathrm{Ca}^{2+}$-gated $\mathrm{K}^{+}$channel, mIK1. Roles in regulatory volume decrease and erythroid differentiation. J Biol Chem 273:2154221553

46. Waisman DM, Gimble JM, Goodman DBP, Rasmussen H (1981) Studies of the $\mathrm{Ca}^{2+}$ transport mechanism of human erythrocyte inside-out plasma membrane vesicles. J Biol Chem 256:409-414 